\title{
Sentinel-site surveillance of Mycobacterium avium complex pulmonary disease
}

\author{
J. Maugein*, M. Dailloux\#, B. Carbonnelle ${ }^{\Uparrow}$, V. Vincent ${ }^{+}$, J. Grosset ${ }^{\S}$ and the French \\ Mycobacteria Study Group
}

ABSTRACT: The incidence of Mycobacterium avium complex (MAC) pulmonary disease in HIVnegative patients was studied prospectively from January 1, 2000 to December 31, 2002 through 32 sentinel sites distributed all over France.

Among the 275 patients who yielded MAC isolates from respiratory clinical specimens, 101 (36.7\%) met the bacteriological, radiographical and clinical criteria established by the American Thoracic Society for nontuberculous mycobacterial respiratory disease. Of these 101 patients, 81 had underlying lung disease, mainly previous tuberculosis, bronchectasis or chronic obstructive pulmonary disease.

Among the 20 patients with no underlying lung disease, 12 had a predisposing factor such as leukaemia or immunosuppressive treatment and eight had no predisposing factor. All patients with MAC respiratory disease had clinical symptoms, commonly cough and fatigue, and 52 (51.5\%) were sputum smear positive for acid-fast bacillus.

The ratio of patients with Mycobacterium avium complex pulmonary disease to patients with pulmonary tuberculosis in France was estimated to be 3\% and the incidence of Mycobacterium avium complex pulmonary disease in France was 0.2 per 100,000 inhabitants.

KEYWORDS: HIV-negative patients, Mycobacterium avium complex, pulmonary disease

$\mathbf{T}$ he nontuberculous mycobacteria (NTM) are environmental organisms commonly isolated from respiratory clinical specimens. They are often contaminants, although the incidence of NTM pulmonary disease is reportedly on the increase in many industrialised countries [1-2]. Among NTM, Mycobacterium avium complex (MAC) is most commonly associated with human disease [1-3]. Pulmonary infection usually occurs in patients with preexisting lung lesions, while disseminated infection mainly occurs in immunocompromised hosts such as patients with AIDS. However, recent studies have reported NTM pulmonary infection in patients without underlying immunosuppressive disorders or chronic pulmonary disease [4-5]. The incidence of NTM infection varies from country to country [6]. In contrast to Mycobactrium tuberculosis, MAC has the potential to contaminate clinical specimens and colonise nonsterile anatomical sites without causing invasive disease. Thus, MAC isolation from a clinical specimen is insufficient to document MAC disease [7]; it is necessary to provide evidence of invasive disease. For this reason, the American Thoracic Society (ATS) has set bacteriological, clinical and radiographical criteria that should be met to document pulmonary disease by NTM [1]. To estimate the current incidence of MAC disease in France, these criteria were applied over a 3-yr period by 32 sentinel sites to all patients without HIV infection from whom an isolate of MAC was obtained.

\section{MATERIALS AND METHODS}

Laboratory and clinical data from all patients who yielded an isolate of MAC in 32 sentinel sites from January 1, 2000 to December 31, 2002 were prospectively collected. The sentinel sites are part of a voluntary-based laboratory network, the French Mycobacteria Study Group (FMSG), whose role is to assure systematic surveillance of M. tuberculosis drug resistance [8]. As shown in figure 1 , the laboratories, which were mainly clinical laboratories of university hospitals, were distributed throughout France, 21 in cities across the country and 11 in the region of Paris.

A definitive diagnosis of MAC infection was made by the recovery of causative organisms from blood or other natural sterile sites (e.g. lymph node, bone marrow). For MAC isolated from respiratory specimens, the bacteriological criteria for diagnosis were a minimum of three positive cultures with negative smears, or two positive cultures with a minimum of one positive
AFFILIATIONS

*Hôpital Haut-Lévêque,

\#Hôpital Brabois,

"Hôpital CHU,

+Institut Pasteur and

${ }^{\S}$ Hôpital Pitie-Salpetriere, Paris,

France.

CORRESPONDENCE

J. Maugein

Laboratoire de Bactériologie

Hôpital Haut Lévêque

Avenue Magellan

33604 Pessac

Bordeaux

France

Fax: 33557656867

E-mail: jeanne.maugein@chu-

bordeaux.fr

Received:

December 282004

Accepted after revision:

August 082005 


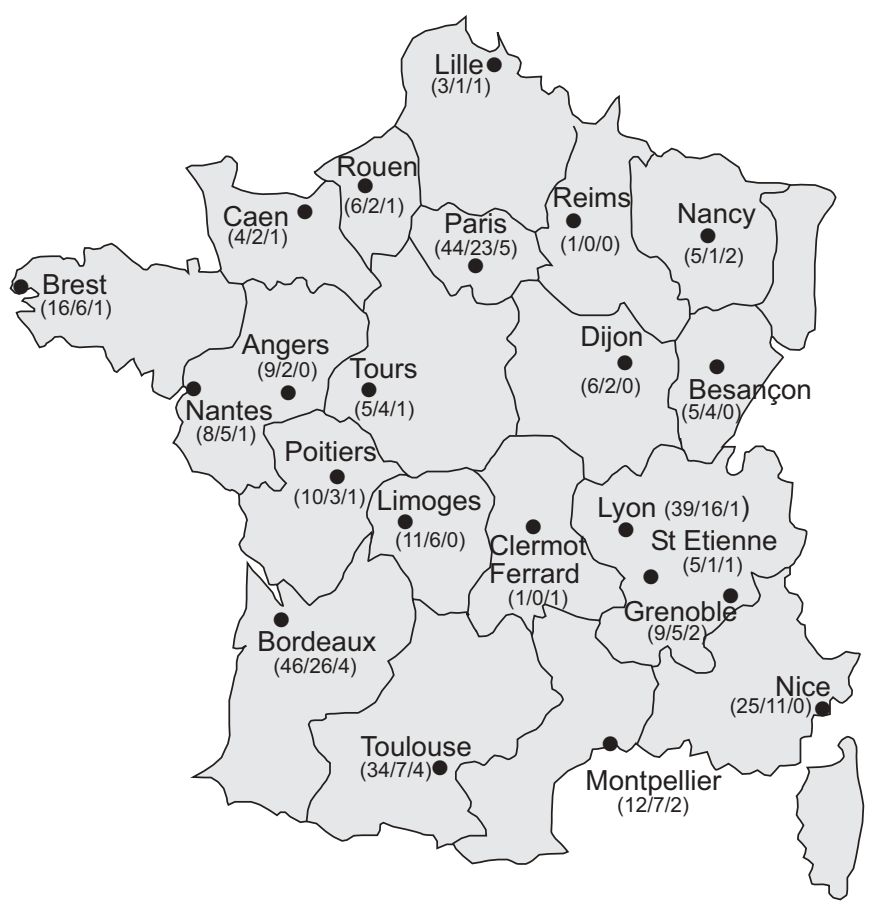

FIGURE 1. Geographical distribution of the sentinel sites of the French Mycobacteria Study Group. Data presented as number of strains/pulmonary disease/extrapulmonary strains.

smear [1]. If sputum samples could not be obtained, a single positive culture from a bronchial washing was sufficient, provided there was a minimum of two (10-99 colonies) growths or a positive smear. For a definitive diagnosis of MAC pulmonary disease [1], the clinical criterion to be met was documented deterioration, in cases of underlying condition, while the radiographical criterion was evidence of progression, if baseline films $>1$-yr old.

Whenever a strain of MAC was isolated, a "case report form" was filled in containing the patient's demographic data, bacteriological criteria (number of positive and negative specimens, acid-fast bacillus (AFB) smear) and, if ATS bacteriological criteria were met, data on clinical symptoms (cough, fatigue, fever, weight loss, haemoptysis, dyspnoea), HIV status, pre-existing lung disease, history of previously treated tuberculosis, underlying illnesses, chest radiographical findings at the time the sputum sample, and past and present drug treatment. Patients were excluded from the study if they were HIV positive.

Cultures of specimens were performed in all laboratories by plating decontaminated specimens onto Löwenstein-Jensen medium (Bio-Rad, Marnes La coquette, France), and in some of the laboratories by inoculation into broths such as MGIT (Mycobacteria Growth Indicator Tube; Becton Dickinson, NJ, USA) or 7H12 (Bactec TB; Becton Dickinson). Putative MAC isolates were identified using DNA probes (GenProbe Inc, San Diego, CA, USA). In several laboratories, organisms identified as MAC using the DNA probe were then tested with additional probes to distinguish $M$. avium from Mycobacterium intracellulare. Some laboratories used INNOLiPA Mycobacteria (Innogenetics, Zwijnaarde, Belgique).
To evaluate the relative incidence of MAC pulmonary disease, each laboratory provided the number of patients with culturepositive tuberculosis during the same period of surveillance.

\section{RESULTS}

\section{Patients with Mycobacterium avium complex infection}

Between January 1, 2000 and December 31, 2002, the FMSG sentinel sites identified 304 patients with MAC isolates, 275 from respiratory specimens and 29 from nonrespiratory specimens. Table 1 gives the distribution of MAC isolates by origin of specimens and potential clinical significance in adult and paediatric $(<15 \mathrm{yrs})$ patients. Among the 275 patients with MAC isolates from respiratory specimens, 134 (48.7\%) met the ATS bacteriological criteria for pulmonary disease while 59 $(21.4 \%)$ had isolates that were considered contaminants or transient colonisation, and $82(29.8 \%)$ could not be included because of insufficient numbers of clinical specimens with positive cultures [1]. Pulmonary disease was most frequent in females, 80 of $134(59.7 \%)$. There was no age difference between patients who met criteria for MAC disease and those who did not, the median age at diagnosis being close to $70 \mathrm{yrs}$ (range 1-98 yrs). However, smear-positive specimens were significantly more frequently obtained from patients who met criteria for MAC disease $(48.5 \%)$ than from those who did not $(5 \%)$. Among clinical specimens from patients excluded because of insufficient number of clinical specimens, $12 \%$ were AFB smear positive. $M$. avium was the most commonly observed subspecies, but the proportion of $M$. intracellulare subspecies was higher among patients with MAC "pulmonary disease" $(41.5 \%)$ than among patients with MAC "contamination or colonisation" (30\%). Data obtained from patients excluded because of an insufficient number of clinical specimens suggests that some could have been considered as having pulmonary disease if more clinical specimens had been obtained.

Information on clinical and radiological conditions was available for 119 of the 134 patients who met the ATS bacteriological criteria. All of them had clinical symptoms and 117 had radiographical abnormalities. The most common clinical symptoms were cough (78\%) and fatigue $(59 \%)$, followed by dyspnoea and weight loss (49\%), fever (27\%)

\begin{tabular}{|c|c|c|c|c|c|c|}
\hline \multirow{2}{*}{$\begin{array}{l}\text { Origin of clinical } \\
\text { specimens }\end{array}$} & \multicolumn{3}{|c|}{ Adults } & \multicolumn{3}{|c|}{ Children } \\
\hline & 2000 & 2001 & 2002 & 2000 & 2001 & 2002 \\
\hline \multicolumn{7}{|l|}{ Respiratory } \\
\hline Pulmonary disease & 48 & 40 & 44 & & 2 & \\
\hline $\begin{array}{l}\text { Contamination or } \\
\text { colonisation }\end{array}$ & 31 & 18 & 9 & 1 & & \\
\hline Doubtful & 39 & 30 & 12 & & 1 & \\
\hline Lymphadenitis & 2 & 2 & 1 & 6 & 3 & 2 \\
\hline Disseminated & 2 & 1 & & & & \\
\hline Others & 2 & 3 & 4 & 2 & & \\
\hline
\end{tabular}


and haemoptysis (15\%). Haemoptysis was the only clinical sign in $3 \%$ of cases. Pulmonary infiltrates were the most common chest radiograph feature (36 patients), followed by nodules (13 patients), infiltrates combined with nodules (8 patients) and cavities ( 8 patients). Among the 117 patients with lung abnormalities, 16 did not have deteriorating infiltrates compared with previous chest radiographs and were not considered to have met the radiographical criteria for MAC pulmonary disease. Therefore, the number of identified patients who met all bacteriological, clinical and radiographical ATS criteria was 101. Among these, 81 (80\%) had underlying lung disease. Previous tuberculosis, bronchiectasis and chronic obstructive pulmonary disease were the most common conditions, present in 33, 28 and 16 patients respectively. Two children had cystic fibrosis. Finally, there was no previous pulmonary disease in $22(22 \%)$ patients, of whom 12 had a predisposing factor for MAC disease (leukaemia, immunosuppressive treatment). Of those without a predisposing factor, seven of the 10 were females who were aged $50,60,72,79,82$, 85 and 88 yrs respectively.

\section{Relative prevalence of Mycobacterium avium complex disease in France}

During the 3-yr study period, 101 patients were identified by the FMSG as having MAC respiratory disease (table 2). During the same time period the FMSG sentinel sites identified 4,766 culture-positive patients with pulmonary tuberculosis. From these findings, the incidence of MAC pulmonary disease among HIV-negative individuals in France could be estimated. Considering that 101 cases of respiratory MAC disease have been detected in a 3-yr period, the annual, average number of cases is $33.6(101 / 3)$. Assuming that the prevalence of tuberculosis cases in France is 11 per 100,000 [9], the annual population studied by the FMSG is close to 14 million ((4766/ $3) / 11 \times 100,000=14.4$ million). Thus, the prevalence of MAC disease will be 34 cases for 14.5 million inhabitants or 0.23 per 100,000 inhabitants. Of course, this ratio is lower in areas where the incidence of tuberculosis is higher; for example, $1.2 \%$ in the Paris area and between $5.7-6.6 \%$ in all other parts of France where the incidence of tuberculosis is lower.

\section{DISCUSSION}

The results of the reported sentinel-site surveillance show that $101(36.7 \%)$ of 275 MAC isolates from respiratory specimens could definitely be related to MAC pulmonary disease according to ATS criteria [1]. Only a limited number of other MAC isolates (for example 10 that were associated with AFB-positive smears) could have been added to the isolates related to MAC pulmonary disease if more clinical specimens had been obtained. Overall, the annual incidence of MAC respiratory disease in France could be estimated at 0.23 per 100,000 inhabitants. It is important to emphasise that MAC pulmonary disease is not a reportable condition and that the expertise needed to identify such a condition is limited. It was, therefore, neither advisable nor possible to conduct a standard epidemiological survey based on representative sites. For the present time, the reported incidence is, therefore, the most reliable estimation that the current sentinel-site investigation could provide.

Although MAC pulmonary disease is not reportable in most countries and standard information is not available, other investigators [10-11] have found relatively similar proportions of MAC isolates to be associated with disease. For example, the Centers for Disease Control and Prevention [12] reported on NTM isolates in the USA between 1993-1996, and found that there were $34,633 \mathrm{MAC}$ isolates (i.e. $8,658 \cdot \mathrm{yr}^{-1}$ on average). During the same period [1] the prevalence of MAC pulmonary disease was estimated to be 1.1 per 100,000 population for the entire USA. Even though the total number of reported MAC isolates was emphasised "not to represent the absolute occurrence and distribution of species" [12], the ratio of MAC pulmonary disease on total MAC isolates was $33 \%$, an estimate closer to that observed in the current study. In southwest Ireland, over a 14-yr period from 1987-2000, 36 (20.5\%) of the 175 NTM isolates from clinical specimens were MAC. Among them, 13 were considered contaminant/colonising respiratory isolates and $23(64 \%)$ were disease-causing isolates, of which 14 were from pulmonary specimens [13]. Out of these 14, two were collected from HIV-positive patients, thus leaving 12 MAC pulmonary diseases, although the authors did not provide the exact number of MAC respiratory isolates. In a 6-yr study conducted in Switzerland, only $21 \%$ of HIV-negative individuals with MAC isolates had clinical disease [2]. In a study [14] conducted during 1994 among patients with cystic fibrosis at 21 USA centres, MAC was isolated from 118 subjects but only 25 of them (21\%) met the ATS microbiological criteria for nontuberculous mycobacterial pulmonary disease.

TABLE 2 Bacteriological characteristics of patients who met American Thoracic Society (ATS) criteria

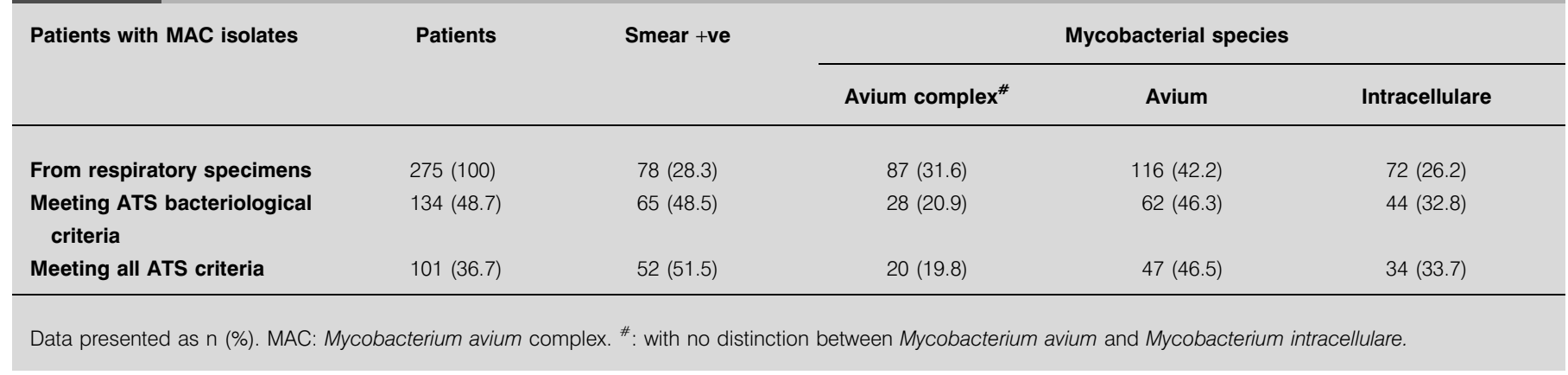


It is important to note that smear-positive clinical specimens were obtained from $48 \%$ of the current study's patients with MAC pulmonary disease and only $2-5 \%$ of patients without definitive MAC pulmonary disease. A similar observation was made by DEBRUNNER et al. [2]. A positive smear is, therefore, an important criterium for the diagnosis of MAC-related disease as stated in the ATS diagnostic criteria [1].

With the exception of those with cystic fibrosis, patients with chronic lung disease due to MAC are generally older adults with a history of underlying chronic lung disease [1]. The current study's patients were no exception to this rule. Their mean age was $70 \mathrm{yrs}$, they were predominantly female, and most of them had pre-existing destructive or obstructive pulmonary conditions, as in the study by KENNEDY and WEBER [15]. Like those of TIERSTEIN et al. [16], some of the current patients had solitary nodules but chronic bronchitis, bronchiectasis and tuberculosis-like infiltrates were the most common features.

Although the majority of the 101 patients in the present study had a history of bronchopulmonary disease, 22 of them had no previous lung disease. Among these 22, 12 had a predisposing factor for MAC disease. Of those without a predisposing factor, seven of the 10 were ageing females who may have shared similarities with the older females who had MAC infection in the absence of pre-existing lung disease, as reported by PRINCE et al. [5]. REICH and JOHNSON [17] coined the term "Lady Windermere Syndrome," a condition affecting female patients with MAC pulmonary disease localised to the middle lobe or the lingual. HUANG et al. [4] confirmed these findings and raised the possible issue of a specific immune deficiency.

In the current study, the incidence of Mycobacterium avium complex pulmonary disease was estimated at 0.2 cases per 100,000 . In southwest Ireland, it was estimated at 0.3 cases per 100,000 [13]. In Switzerland, the incidence of respiratory disease due to all species of nontuberculous mycobacteria was estimated between $0.4-0.9$ per 100,000 during the period 1983-1988 [2]. This case rate is lower than that in the USA where the case rate in HIV-negative patients was estimated at $1.1-1.2$ cases per $100,000[1,18]$. The current study's case rate may be an underestimate, as $30 \%$ of patients could not be classified due to insufficient numbers of clinical specimens. But even if all of these patients had been considered definitive cases of Mycobacterium avium complex respiratory disease, the overall incidence would remain between $0.2-0.3$ per 100,000 . Because of the increasing frequency of Mycobacterium avium complex pulmonary disease [19] and the difficulty of its treatment, it seems advisable to make it a reportable condition based on reasonable microbiological, radiographical and clinical criteria as those recommended by American Thoracic Society.

\section{ACKNOWLEDGEMENTS}

The members of the French Mycobacteria Study Group are as follows: M.L. Abalain (Brest), R. Bauriaud (Toulouse), P. Bemer (Nantes), A. Bourgoin (Poitiers) L. Brasme (Reims), E. Cambau (Paris), A. Carricajo (Saint Etienne), C. Carriere (Montpellier), B. Cattier (Tours), S. Coignard (Paris), M. Chomarat (Lyon), G. Couetdic (Besançon), M.F. David (Paris), L. Deforges
(Creteil), F. Doucet-Populaire (Versailles), J.M. Duez (Dijon) M.C. Gutierrez (Paris), J.L. Herrmann (Paris), V. Jarlier (Paris), F. Jaureguy (Avicennes), V. Lalande (Paris), L. Landraud (Nice), L. Lebrun (Paris), N. Lemaitre (Lille), C. Lorain (Nancy), B. Malbruny (Caen), C. Martin (Limoges), C. Offredo (Paris), M. Pestel-Caron (Rouen), G. Richalet (Grenoble), J. Robert (Paris), J.P. Romaszko (Clermont Ferrand), E. Ronco (Garches), A. Rossier (Paris), D. Sicard (Nice), C. Truffot-Pernot (Paris), A. Vachée (Roubaix), V. Vincent (Paris).

\section{REFERENCES}

1 Wallace RJ Jr, Glassroth J, Griffith DE, Olivier KN, Cook JL, Gordin F. Diagnosis and treatment of disease caused by nontuberculous mycobacteria. Am J Respir Crit Care Med 1997; 156: S1-S25.

2 Debrunner M, Salfinger M, Brändli O, Von Graevenitz A. Epidemiology and clinical significance of nontuberculosis mycobacteria in patients negative for human immunodeficiency virus in Switzerland. Clin Infect Dis 1992; 15: 330-345.

3 Olivier KN. Nontuberculous mycobacterial pulmonary disease. Curr Opin Pulm Med 1998; 4: 148-153.

4 Huang JH, Kao PN, Adi V, Ruoss SJ. Mycobacterium aviumintracellulare pulmonary infection in HIV negative patients without preexisting lung disease. Chest 1999; 115: 1033-1040.

5 Prince DS, Peterson DD, Steiner RM, et al. Infection with Mycobacterium avium complex in patients without predisposing conditions. $N$ Engl J Med 1989; 321: 863-868.

6 O'Brien RJ, Geiter LJ, Snider DE. The epidemiology of nontuberculous mycobacterial diseases in the United States. Results from a national study. Am Rev Respir Dis 1987; 135: 1007-1014.

7 Kanathur N, Shantaveerapa HN, Byrd RP, Mehta JB, ROYTM. Nontubercular mycobacterial pulmonary infection in immunocompetent men. Southern Med J 2001; 94: 719-723.

8 Robert J, Jarlier V. Tuberculose et resistance aux antituberculeux. Surveillance nationale des maladies infectieuses. [Tuberculosis and antituberculosis drugs resistence. National survey of infectious diseases]. Institut de veille sanitaire (InVS) 2002; 239-243.

9 Decludt B, Campese C. Les cas de tuberculose déclarés en France en 1998 et 1999. [Cases of tuberculosis reported in France between 1998 and 1999]. Bull Epidemiol Hebdo 2001; 10: 41-43.

10 Good RC, Snider DE. Isolation of nontuberculous mycobacteria in the United States. J Infect Dis 1980; 146: 829-833.

11 Wickman K. Clinical significance of nontuberculous mycobacteria. Scand J Infect Dis 1986; 18: 337-341.

12 Butler WJ, Crawford JT, Shutt K. Nontuberculous mycobacteria reported to the Public Health laboratory information system by state public health laboratories, United States, 1993-1996. NTM Report, CDC, Atlanta, 1999. 
13 Kennedy MP, O'Connor TM, Ryan C, Sheehan S, Cryan B, Bredin C. Nontuberculous mycobacteria: incidence in southwest Ireland from 1987 to 2000. Respir Med 2003; 97: 257-263.

14 Olivier KN, Weber DJ, Wallace RJ, et al. Nontuberculous mycobacteria in cystic fibrosis study group. Am J Respir Crit Care Med 2003; 167: 810-812.

15 Kennedy TP, Weber DJ. Nontuberculous mycobacteria. An underappreciated cause of geriatric lung disease. Am J Respir Crit Care Med 1994; 149: 1654-1658.

16 Teirstein AS, Damsker B, Kirschner PA, Krellenstein DJ, Robinson B, Chuang MT. Pulmonary infection with MAI: diagnosis, clinical patterns, treatment. Mt Sinai J Med 1999; 57: 209-215.

17 Reich JM, Johnson RE. Mycobacterium avium complex pulmonary disease presenting as an isolated lingular or middle lobe pattern: the Lady Windermere syndrome. Chest 1992; 101: 1605-1609.

18 Wolinsky E. Mycobacterial diseases other than tuberculosis. Clin Infec Dis 1992; 15: 1-12.

19 Maras TK, Daley CL. Epidemiology of human pulmonary infection with nontuberculous mycobacteria. Clin Chest Med 2002; 23: 553-567. 